\title{
Detection of secondary electrons in a multidipole plasma
}

\author{
Noah Hershkowitz, R. L. Goettsch, and Chung Chan \\ Department of Nuclear Engineering, University of Wisconsin, Madison, Wisconsin 53706 \\ Kyle Hendricks and R. T. Carpenter \\ Department of Physics and Astronomy, University of Iowa, Iowa City, Iowa 52242
}

(Received 23 December 1981; accepted for publication 5 April 1982)

\begin{abstract}
Secondary electrons are identified as a separate component within a plasma in a specifically modified multidipole device. It is shown that secondary electron emission at walls can be comparable to electron production by ionization. It is also shown that secondary electron emission can significantly change Langmuir probe characteristics.
\end{abstract}

PACS numbers: $52.50 . \mathrm{Dg}, 52.80 .-\mathrm{s}, 52.70 . \mathrm{Nc}$

Basic plasma properties have been studied in filament discharge plasmas for many years. ${ }^{1}$ In such devices, plasma electrons and ions are produced by energetic primary electrons which are emitted from filaments mounted in a vacuum chamber. Primary electron confinement in such discharge plasmas has been significantly improved by the addition of multidipole surface magnetic fields. ${ }^{2}$

Primary electron-induced secondary electron emission currents from plasma boundaries (e.g., walls) with energies less than $10 \mathrm{eV}$ can be comparable to primary electron currents to the boundaries when primary energies are the order of $100 \mathrm{eV} .^{3}$ Significant densities of primary ionizing electrons are usually present in filament discharge devices, especially in multidipole devices ${ }^{2}$ which can be operated at low neutral pressures, so secondary electrons might be expected to be present. Nevertheless, secondary electrons have previously been neglected in discussions of such devices, probably because they cannot be distinguished from plasma electrons which have similar energy spreads $(<10 \mathrm{eV})$. In this paper, we present direct experimental evidence that the secondaries are present in multidipole discharge devices together with plasma and primary electrons, and that they can play an important role in such devices.

The plasma potential in discharge plasmas is normally several plasma electron temperatures $T_{e} / e$, where $e$ is the electron charge, more positive than the walls which serve as the anode. Secondary electrons emitted with an energy spread $\sim T_{e}$ gain only a few $T_{e}$ in accelerating through the wall sheath and cannot be distinguished from plasma electrons. They can, however, be distinguished from plasma electrons if the plasma potential $\Phi_{p}$ (which is positive and uniform, except near the walls) is adjusted so that $e \Phi_{p} / T_{e}$ $>1$. This was accomplished using the $e$-pot device. ${ }^{4}$

The e-pot (for the electrostatic soup pot and named because it is constructed out of a 40-1 cooking pot) device is a conventional stainless steel multidipole discharge device ${ }^{2}$ modified by the addition of pairs of electrodes which serve as anodes, above and parallel to the magnetic cusps (see Fig. 1). The maximum cusp $B$ field was $10^{3} \mathrm{G}$ and the cusp length $L_{\text {c }}$ was $800 \mathrm{~cm}$. The gaps between the pairs of electrodes are located directly over the cusps. The plasma potential tends to follow the electrode potential; i.e., the electrodes serve as effective anodes for the plasma electrons and ions, but pri- mary electrons are magnetically confined to the cusps. Plasma potentials as high as $100 \mathrm{~V}$ can easily be achieved so $e \Phi_{p} / T_{e} \gg 1$ is easily satisfied. Details of this device, which provides electrostatic stoppering of the multicusps, have been given elsewhere. ${ }^{4}$

The density of each of the three species, plasma electrons, primary electrons and secondary electrons, can be $d i$ rectly determined from Langmuir probe current-voltage $(I-$ $\Phi)$ characteristics in multidipole plasmas. The special characteristics of these devices is that primary electrons are confined very well by the surface magnetic field, and make many bounces from the walls before being lost. At each cusp, the effective leak width for primary electrons is known to be the order of the primary electron gyroradius evaluated at the wall. This results in a distribution function for primary electrons which closely resembles a spherical shell in velocity space (i.e., monoenergetic electrons with speed $v$, isotropically distributed). Projecting this velocity distribution into one dimension results ${ }^{2}$ in a "water bag" distribution function, that is

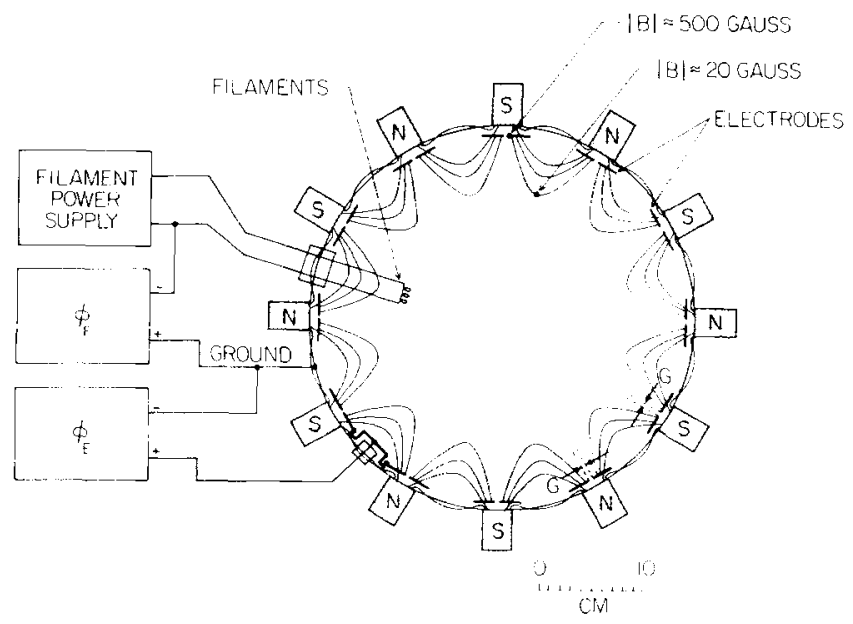

FIG. 1. Schematic of the $e$-pot device. The electrode and magnetic field configuration are shown. Magnetic poles face the plasma and alternate in polarity, as indicated. The magnet rows and electrodes are continued across the top and bottom. 


$$
f\left(v_{x}\right) \propto\left\{\begin{array}{cc}
1, & 0 \leqslant v_{x} \leqslant v_{p} \\
0, & v_{p}<v_{x}
\end{array},\right.
$$

where $v_{p}$ is the monoenergetic primary electron speed.

A linear plot of a current-voltage trace from a Langmuir probe (with area $S$ ) for such a distribution function is a straight line ${ }^{3}$ whose slope $d I / d \Phi$ is proportional to the primary electron density $n_{D}$ :

$$
d I / d \Phi=\left(\frac{1}{4}\right) n_{p} v_{p} e S / E_{p},
$$

where $E_{p} \equiv\left(\frac{1}{2}\right) m v_{p}^{2}$ and $m$ is the electron mass.

This argument also applies to the secondary electrons, as well when plasma potential satisfies $e \Phi_{p} / T_{e}>1$ so an ideal Langmuir probe trace is composed of two straight line portions and a Maxwellian part (corresponding to the plasma electrons).

Before we can extract the saturation currents associated with primary and secondary electrons from the probe characteristics, it is necessary to correct the curves for secondary emission from the probes themselves. We can estimate the contribution of these secondary electrons from the following argument. The probe current can be expressed as

$$
I \propto \int_{v_{\min }}^{\infty} f(v) v\left[1-\delta\left(v_{w}\right)\right] d v,
$$

where $\delta\left(v_{w}\right)$ is the ratio of secondary to primary current to the probe. The velocity of electrons at the probe defined to be $v_{w}$ goes to zero when $v=v_{\min }$. Energy conservation gives $v^{2}+2 e \Phi / m=v_{w}^{2}$ so $v_{\min }=(-2 e \Phi / m)^{1 / 2}$, where $\Phi$ is the probe bias voltage. Considering the contribution of secondary electrons at the probe due to only primary electrons, we can take the $f(v)$ given by Eq. (1). For simplicity, we approximate $\delta$ by

$$
\delta\left(v_{w}\right) \approx v_{w}^{2} / v_{u}^{2},
$$

where the constant $v_{u}$ is defined by $\delta=1$ when $v_{w}=v_{u}$. Substituting into Eq. (3) and defining $E_{u} \equiv\left(\frac{1}{2}\right) m v_{u}^{2}$ gives

$$
\begin{aligned}
I \propto \int_{v_{\min }}^{v_{p}} v\left(1-\frac{v^{2}+2 e \Phi / m}{v_{u}^{2}}\right) d v \\
\quad=\frac{1}{m E_{u}}\left[\left(E_{p}+e \Phi\right)\left(E_{u}-e \Phi\right)-\left(E_{p}^{2}-e^{2} \Phi^{2}\right) / 2\right] .
\end{aligned}
$$

The primary electron current to the probe, when the probe is biased at the plasma potential $\Phi_{p}$, is the saturation current $I_{p}^{*} \propto\left(E_{p}+e \Phi_{p}\right) / m$.

We could not find recent data for $\delta$ corresponding to tantalum. Comparison of Eq. (4) to tungsten data ${ }^{3}$ (which has similar emission characteristics to tantalum) allows us to identify $E_{u} \approx 150 \mathrm{eV}$. The approximation used in Eq. (4) tends to underestimate $\delta$ slightly and has a maximum error of about 0.15 or $25 \%$ at $E_{p}=70 \mathrm{eV}$. A representative probe trace which exhibits all three components and the effects of secondary electrons from the probe is shown in Fig. 2. The various components used to fit this trace are also shown. The primary current $I_{p}$ is not a straight line because it includes the effects of secondary electron emission from the probe. $I_{s}$ is the secondary electron current from the wall, and $I_{e}$ is the Maxwellian plasma electron contribution. Note that the sa-

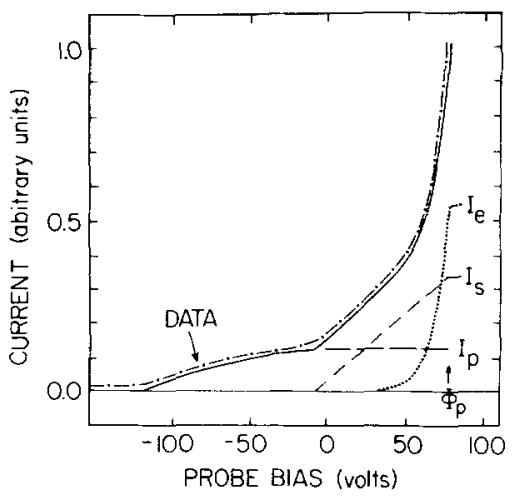

FIG. 2. A representative Langmuir probe trace (labeled DATA). The plasma potential was $81.5 \mathrm{~V}$. Data has been displaced slightly to allow comparison with the fit to the data. The contributions from the plasma electrons $I_{\mathrm{e}}$, primary electrons $I_{\rho}$, and secondary electrons $I_{s}$ are separately indicated. The neutral pressure was $5 \times 10^{-6}$ Torr.

turation currents associated with all three components are comparable, and that the reduction in apparent primary electron current is significant. Results of these fits are not sensitive to the details of the emission coefficient used for the secondary emission at the probe.

We can estimate $\delta$ at the walls from the ratio of secondary to primary probe saturation current. The creation rate of secondary electrons at the walls is given by $\delta n_{p} v_{p} A_{p}$, where $n_{p}$ is the primary electron density, $v_{p}$ is the primary electron velocity at the boundary, and $A_{p}$ is the effective loss area. Secondary electrons are lost to either the walls (with loss area $A_{s}$ ) or to ionization. Secondary electrons lose much more energy to ionization than their initial energy or energy spread (before they were accelerated by the wall sheath). An ionization event prevents them from leaving the plasma along a cusp because they no longer have enough energy to overcome the wall sheath potential. Such electrons thermalize, and are eventually lost by diffusion across the cusp magnetic field to the electrodes. Balancing secondary electron creation and loss gives

$$
\delta n_{p} v_{p} A_{p}=n_{s} v_{s}\left(A_{s}+V \sigma_{i} n_{0}\right),
$$

where $V$ is the plasma volume, $n_{0}$ the neutral density, and $\sigma_{i}$ is the ionization cross section. In terms of probe saturation currents $I^{*} \propto n v$, so Eq. (6) gives

$$
\delta=\frac{I_{s}^{*}}{I_{p}^{*}}\left[\left(A_{s}+V \sigma_{i} n_{0}\right) / A_{p}\right]
$$

The area term $A_{p}$ in the denominator of Eq. (7) can be written as $A_{p}=r_{p} L_{c}$ by assuming that the effective loss area for primary electrons is the product of their gyroradius $r_{p}$ and the cusp length $L_{c}{ }^{3,5}$ Note that $r_{p}$ can be written $r_{p}$ $=2.4\left(2 E_{p}\right)^{1 / 2} / B \approx 3.4 \times 10^{-3}\left(E_{p}\right)^{1 / 2}$ in this experiment, so for example, $A_{p}=27 \mathrm{~cm}^{2}$ when $E_{p}=100 \mathrm{eV}$.

The numerator of Eq. (7) is somewhat more complicated because the relative sizes of the two terms depend on details of the device, the neutral pressure, and on the mechanism by which secondary electrons are lost to the cusps. The effective leak area for secondaries might be as small as $r_{s} L_{c}$, where $r_{s}$ is the secondary electron gyroradius (evaluated at the wall and approximately equal to $8 \times 10^{-3} \mathrm{~cm}$ ). However, 


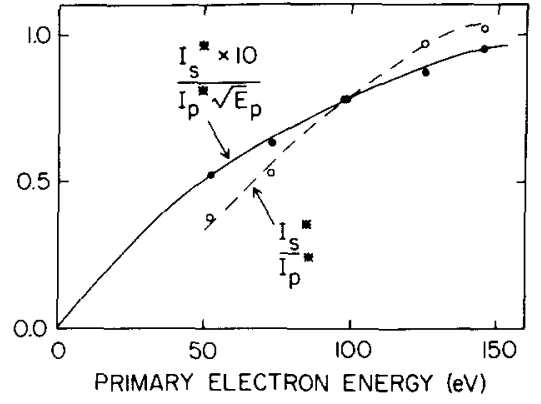

FIG. 3. The ratios $I_{s}^{*} /\left(I_{p}^{*}\right)$ and $10 I_{s}^{*} /\left(I_{p} \vee E_{p}\right)$ vs primary electron energy.

the secondaries have very little energy when they reach the wall so one might expect that they will be strongly influenced by self-consistent electric fields across the cusps at the other extreme. We can get an upper limit to the effective leak area by taking the gap width for the leak width (the order of $1 \mathrm{~cm}$ ). Thus the leak area $A_{s}$ is somewhere between $8 \times 10^{-3}$ $\mathrm{cm} \times L_{c}$ and $1 \mathrm{~cm} \times L_{c}$ (i.e., from 6 to $800 \mathrm{~cm}^{2}$ ). For an argon plasma at neutral pressure $p$ (Torr), the ionization term can be evaluated by taking $V \approx 2.2 \times 10^{4} \mathrm{~cm}^{3}, \sigma_{i} \approx 2.4 \times 10^{-16}$ $\mathrm{cm}^{2}$ (Ref. 4) and $n_{0}=3 \times 10^{16} \mathrm{p}$, where $p$ is measured in Torr, one gets

$$
V \sigma_{i} n_{0} \approx 1.6 \times 10^{5} \mathrm{p} .
$$

For example, at $p=5 \times 10^{-6}$ Torr, this term equals $0.8 \mathrm{~cm}^{2}$. Taking the extreme estimates, the term in brackets can range from 0.2 to 30 . Although $E_{p}$ varied for data in this experiment, $E_{s}$ is determined by the details of secondary emission, and are essentially constant here, so we expect the term in the brackets to be some constant multiplied by $1 / V E_{p}$.

Experimental values of $I_{s}^{*} / I_{p}^{*}$ determined from data, similar to those given in Fig. 2, are given in Fig. 3. Note that values of the ratio $I_{s}^{*} / I_{p}^{*}$ were comparable to 1.0 so that even the minimum value for the bracket in Eq. (7), i.e., 0.2, gives $\delta$ as large as 0.2 . If we divide these data by $\sqrt{ } E_{p}$ the resulting data should be proportional to $\delta$. These data, also given in Fig. 3, show that $\delta$ is generally proportional to the primary energy, and that the shape is as expected for this energy range.

Since secondary electrons from the walls have not been emphasized in descriptions of previous multidipole devices, it is of interest to estimate their contribution to our plasma. The production rate of secondary electrons can be approximated by $\delta n_{p} v_{p} A_{p}$, while the production of plasma electrons by primary electrons is roughly $n_{p} v_{p} \sigma_{i} n_{0} V$ (neglecting the energetic electrons, which are degraded in energy by ionization). The ratio of secondary electron production rate to plasma electron production rate is then $\delta A_{p} / V \sigma_{i} n_{0}=34 \delta$ at $p=5 \times 10^{-6}$ Torr. Taking even the minimum value of $\delta=0.2$ gives $\delta A_{p} / V \sigma_{i} n_{0}=6.8$, so many more electrons are being produced by secondary emission than by ionization.

In the $e$-pot device plasma, electrons are electrostatically confined at the cusps, and losses occur by diffusion across the cusps ${ }^{4}$ while secondaries are not electrostatically confined, unless they loss energy. Thus the ratio of density of plasma electrons to secondary electrons in e-pot can be quite high, and their probe saturation currents can be comparable, as seen in Fig. 2. In conventional multidipole devices ${ }^{2}$ where $e \Phi_{p} / T_{e} \approx 1$, the confinement of plasma and secondary electrons is essentially the same, and the factor of 6.8 becomes even more important.

Electron temperatures in the $e$-pot device at low neutral pressure were found to be higher than those reported previously ( $10 \mathrm{eV}$ compared to $5 \mathrm{eV}$ at $5 \times 10^{-6}$ Torr in argon). About half of this increase in temperature can be attributed to improved confinement in the device itself, while the other half comes from properly including the secondary electron contribution to the probe current. This latter effect has not been taken into consideration previously.

Secondary electrons have not been considered in previous multidipole experiments because they are indistinguishable from plasma electrons when wall sheaths are small. In addition, at high neutral pressures, primary electron densities are relatively low. The presence of secondary electrons is not negligible for low neutral pressure regimes. Calculations of particle balance and energy balances in multidipole devices, that do not take secondaries into account, can result in significant errors. Secondary electrons are also important in interpreting Langmuir probe data. It is apparent that the secondary electrons emitted from the probe can lead to substantial misjudgment of the primary electron saturation current, and, consequently, the plasma electron temperature.

We are grateful to J. R. DeKock for technical assistance throughout this experiment. We thank Tony Leonard for help in data-taking and analysis in the early stages of this experiment. This work was supported by the U.S. Department of Energy Contract DE-AC02-76ET53034.

'I. Alexeff, W. D. Jones, and K. E. Lonngren, Phys. Rev. Lett. 21, 1968. ${ }^{2}$ K. N. Leung, N. Hershkowitz, and K, R. MacKenzie, Phys. Fluids 19 , 1045 (1976); K. N. Leung, T. K. Samec, and A. J. Lamm, Phys. Lett. A 51, $490(1975)$.

${ }^{3} \mathrm{G}$. Hachenberg and W. Brauer, Aduances in Electronics and Electron Physics XI, edited by L. Marton (Academic, New York, 1959), p. 413; M-C. Saussez-Hublet and P. J. Harbour, Secondary Electron Emission of Sapphire, Tungsten, Molybdenum and Titanium for Maxwellian Incident Electrons, Culham Report CLM-R 208, 1980.

${ }^{4}$ N. Hershkowitz, K. Hendricks, and R. T. Carpenter, J. Appl. Phys. 53, 4105 (1982).

${ }^{5}$ Knorr, G. and Goertz, C. K., Astrophys. Space Sci. 31, 209 (1974). 